\title{
A Study on the Control Technique of Construction Project Process
}

\author{
Changming Hu, Shaoping Zhang, Xueyan Wang \\ College of Civil Engineering, Xi’an University of Architecture \& Technology, Xi’an, China \\ Email: hu.tm@163.com
}

Received January 15, 2013; revised February 16, 2013; accepted February 23, 2013

\begin{abstract}
The construction process control of large-scale projects is one of the difficulties and the keys in the owner's project management. Based on generalization process index, this paper proposes an approach to construction project process control. The paper elaborates on the concept and calculation of generalization process index, which, on analysis, possesses Markov property. Monthly generalization process index is regarded as a state of Markov chain, and the transition between different states is realized by computer simulation. According to the Markov process forecasting model and by means of MATLAB program, the project process forecast is realized, and the completion probability in contract period is obtained. From practical instances, it is concluded that this approach has good applicability and operability and that the obtained results can reflect the degree of project risks.
\end{abstract}

Keywords: Generalization Process Index; Markov Computer Stimulation; Project Process Control

\section{Introduction}

Large-scale projects are usually highly professional and independent, with long construction period, complex engineering structure and complex construction technique, as well as many cooperative building units. The process management of such projects directly affects the qualities of the projects and the production of enterprises. At present, the large-scale project management organizations of most Chinese large enterprises usually employ such traditional methods as comparison of horizontal diagram, comparison of S-shaped curve process, comparison of "banana"-shaped curve. As is indicated by practice, with the methods above, the project management is strict and inflexible, which disadvantages the contractor and increases the owner's management load. Therefore, with the premise of set quality and schedule, a more applicable project process control approach ought to be sought, in order to overcome the defects of the traditional methods in satisfying practical engineering needs, and to guarantee management at a higher level.

Process control model based on generalization process index can provide a new method for schedule control. By this method the owner can grasp overall progress macroscopically and know the risk of engineering project. Its guidelines are as follows: first, the concept of generalization process index is to put forward; second, the generalization process index, on analysis, possesses Markov

*Foundation item: Xi’an social science planning project issue (11J058). property; third, a Markov stimulated process control model, based on generalization process index, is established; therefore, the completion probability in contract period is obtained.

\section{Concept and Calculation of Generalization Process Index}

\subsection{Concept of Generalization Process Index}

Generalization process index means the sum of the percentages of the completed quantities (plan or actual) of a project's separated parts that account for the total quantities at a specified time, noted as GPI (generalization process index). If the quantities are plan quantities, the generalization process index is called plan generalization process index; if the quantities are actual quantities, actual generalization process index [1,2].

$$
\mathrm{GPI}=\sum_{i=1}^{n} \frac{P A_{i}}{\mathrm{TPA}}
$$

Type: $P A_{i}$ - the plan or actual completed quantities of a project's separated parts at a specified time;

TPA - total quantities of a project;

$n$-sum of total process at a specified time.

\subsection{Calculation of Generalization Process Index [3]}

GPI has been widely used in Baosteel technological 
transformation project. Take Baosteel 2\# color coating project as an example to demonstrate the calculation of GPI. In March 2001, three tasks need to be finished in the project-a part of pile foundation, a part of reinforced concrete of pile foundation, a part of steel structure fabrication.

1) According to past experience (the main reference is the manual work needed to complete a physical quantity), each physical quantity and important work are turned into percentage. The pile, reinforced concrete and steel structure account respectively for $7 \%, 10 \%, 10 \%$ of the total.
2) According to the construction contract schedule and actual completed quantities, the plan and actual completed GPI can be calculated monthly. The project schedule and actual condition are shown in Table 1. Suppose the decomposition of physical quantities is evenly distributed in this project when GPI is calculated.

The GPI calculation is as follows:

1) There are 992 sets of pile foundation, $15,533 \mathrm{~m}^{3}$ of reinforced concrete and $1926 \mathrm{~T}$ of steel structure in the color coating project.

2) The plan completed GPI of pile foundation in

$$
\begin{aligned}
\text { March } & =\frac{\text { pile foundation quantity of the main factory buildings }}{\text { total pile quantity in the project }} \\
& \times \frac{1}{\text { months needed to complete the pile foundation of the main factory buildings }} \\
& \times \text { the persentage of the pile foundation quantity } \\
& =\frac{776}{992} \times \frac{1}{776 / 163} \times 7 \%=1.15 \%
\end{aligned}
$$

The actual completed GPI of pile foundation in

$$
\begin{aligned}
& \text { March }=\frac{\text { monthly completed pile foundation quantity }}{\text { total pile quantity in the project }} \times \text { the persentage of the pile foundation quantity } \\
& =\frac{150}{992} \times 7 \%=1.059 \%
\end{aligned}
$$

Also available are the plan completed GPI of reinforced concrete in March $=0.258 \%$.

The actual completed GPI of reinforced concrete in March $=0.193 \%$.

The plan completed GPI of steel structure in March = $1.758 \%$.

The actual completed GPI of steel structure in March = $1.588 \%$.

3) The plan completed GPI in March $=1.15 \%+$ $0.258 \%+1.758 \%=3.166 \%$.

The actual completed GPI in March $=1.059 \%+$ $0.193 \%+1.588 \%=2.84 \%$.

The calculation of the actual completed GPI in March is relatively simple. It can be obtained by the sum of the percentages of physical quantities multiplied by the divisor of monthly completed quantities and the total quantities.

4) In accordance with the method above, the plan and actual GPI of the whole project in each month can be obtained.

\section{Establishment of Process Control Model}

\subsection{Analysis of Markov Property in GPI}

The theory of micro Markov process researches the states of a system (e.g. a region, an enterprise, etc.) and their transitions. By the research of the initial probabilities of

Table 1. The project schedule and GPI.

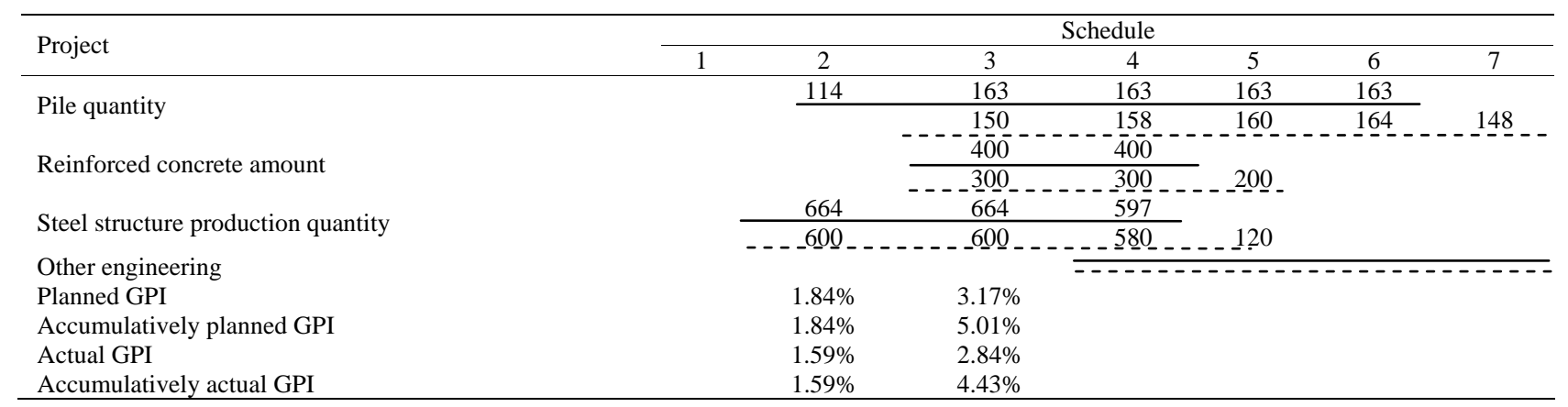


different states and their transition probabilities, the tendency of the changes in states is decided, hence forecast of future state. Markov process is of no aftereffect, which means if the state at the moment of $t_{m}$ is known, the probability property of the state after $t_{m}$ is only related to the state at $t_{m}$, rather than the state before $t_{m}$ [4].

As to a large-scale project, GPI's change is a random variable that depends on $t$ along with the progress of the project, which is a random process. GPI's state at $\tau$ ( $\tau$ $>t$ ) is only related to the state at $t$, rather than the state before $t$. Therefore, the state transition of GPI possesses Markov property. The next month's GPI is only related to this month's GPI, rather than the GPI before this month. Because GPI produces only a finite state (namely possible value) $a_{1}, a_{2}, \cdots$ and GPI transforms its state only at a finite time $t_{1}, t_{2}, \cdots$ So GPI change is a Markov chain [5].

\subsection{Stimulation of Markov Process [6]}

Suppose $\{X(t), t=0,1,2, \cdots\}=\{X(0), X(1), X(2), \cdots\}$ is a Markov chain. The computer stimulation of Markov chain means that a sample function of Markov chain, namely a sample sequence $\left\{a_{0}, a_{1}, a_{2}, \cdots\right\}$, in which $a_{i}$ is the sample value of the random variable $X(i)$, is obtained by the production of $[0,1]$ evenly distributed random numbers and the transition of probability models. Therefore, a sample sequence of Markov chain is obtained as long as the sample value $a_{i}$ is obtained. If $X(0), X(1), \cdots$ are mutually independent and the distribution is known, in accordance with the method of random variable simulation, the sample value $a_{i}$ of each random variable $X(i)$ can be obtained independently. However, random variables of Markov chain are not independent; instead, they are related to each other; that is, they are of Markov property. The relation, nevertheless, is not close, which only requires that the state of any moment is only related to that at preceding moment, namely $X(1)$ is related to $X(0), \cdots, X(i)$ is related to $X(i-1) \ldots$ Therefore, as long as the initial state $a_{0}$ (or the initial distribution) and one-step matrix of transition probabilities $P$ are given, the sample value $a_{1}$ (state) of $X(1)$ can be calculated by time sequence stimulation, hence $a_{2}$ of $X(2)$ by $X(1)=a_{1}$ and $P \ldots$ The rest is inferred until a satisfactory solution is obtained.

If Markov chain $\{X(t), t=0,1,2, \cdots, n\}$ meets the requirements that the initial state is $a_{0}$ and the state space is $E=(0,1,2, \cdots, n)$, the one-step matrix of transition probabilities is

$$
\boldsymbol{P}=\left[\begin{array}{cccc}
P_{00} & P_{01} & \cdots & P_{0 n} \\
P_{10} & P_{11} & \cdots & P_{1 n} \\
\vdots & \vdots & \ddots & \vdots \\
P_{n 0} & P_{n 1} & \cdots & P_{n n}
\end{array}\right]
$$

Its simulation is as follows:

1 ) It is known that the initial state of $X(0)$ is $a_{0}$, namely the sample value of $X(0)$ is $a_{0}$. If the initial state is uncertain, the $[0,1]$ evenly distributed random number $r_{0}$ is produced by its initial distribution,

$$
Q=\left(q_{0}, q_{1}, \cdots, q_{n}\right),
$$

and if, to $a_{0}$, there is

$$
\sum_{j=0}^{a_{0}-1} q_{j}<r_{0} \leq \sum_{j=0}^{a_{0}} q_{j}
$$

the initial state of $X(0)$ is $a_{0}$.

2) If $X(0)=a_{0}, a_{1}$, the sample number of $X(1)$, is to be solved. The distribution of $X(1)$ is known, that is $\left(P_{a_{0} 0}, P_{a_{0} 1}, \cdots, P_{a_{0} n}\right)$, the elements of the line $a_{0}$ in the transition matrix $\boldsymbol{P}$. The first transition state is obtained by $r_{1}$, the $[0,1]$ evenly distributed random number. If, to $a_{1}$, there is

$$
\sum_{j=0}^{a_{1}-1} q_{a_{0} j}<r_{1} \leq \sum_{j=0}^{a_{1}} q_{a_{0} j}
$$

the initial state of $X(1)$ is $a_{1}$. Hence, the state $a_{0}$ transfers to the state $a_{1}$, the sample value of $X(1)$.

3) If $X(1)=a_{1}, a_{2}$, the sample value of $X(2)$, is to be solved. It is known that the distribution of $X(2)$ is $\left(P_{a_{1} 0}, P_{a_{1} 1}, \cdots, P_{a_{1} n}\right)$, the elements of the line $a_{1}$ in the transition matrix $\boldsymbol{P}$. Then the second transition state is obtained by $r_{2}$, the $[0,1]$ evenly distributed random number. If, to $a_{2}$, there is

$$
\sum_{j=0}^{a_{2}-1} q_{a_{1} j}<r_{2} \leq \sum_{j=0}^{a_{2}} q_{a_{1} j}
$$

the initial state of $X(2)$ is $a_{2}$. Hence, the state $a_{1}$ transfers to the state $a_{2}$, the sample value of $X(2)$.

A set of sample values, $a_{0}, a_{1}, a_{2}, \cdots$ of the Markov chain $\{X(0), X(1), X(2), \cdots\}$ are obtained by the above method.

\subsection{Markov Stimulated Process Forecasting Model Based on GPI}

According to the concept of GPI proposed and realized above, the analysis of Markov property, and the description of Markov process stimulation mentioned above, the Markov forecasting model has been established on the completion probability of GPI. The steps are as follows:

1) Determination of state space. According to the size of GPI span, it can be divided into several intervals. Each interval is a state, marked $1,2,3, \cdots$. If $f(x)$ is the forecasting state, then $f(x)$ is a finite state Markov chain. The types of a project state determine its state transition space $\mathrm{E}(1,2,3, \cdots)$.

2) Determination of initial state. Suppose the GPI in the first month after the project starts is in the state, marked 1.

3) Determination of one-step transition matrix. The 
supervisors and the experts of construction unit, as well as the engineers gathered by the owner discuss the onestep transition matrix, which may be modified on a small scale in the following practice.

4) Prediction of monthly GPI. The GPI and the state transition matrix in one month are known; the GPI in the next month can be predicted.

If the state in the first month $a_{1}=1$ is known, the state in the second month $a_{2}$ is to be solved. According to the above method of Markov simulation, $r_{2}$ is to be determined. The GPI in this month, based on $r_{2}$ in the state interval $\left[b_{i-1}, b_{i}\right]$, can be determined with linear interpolation. The GPI in this month $S=b_{i-1}+\left(b_{i}-b_{i-1}\right) \times r$ is obtained by the production of $[0,1]$ evenly distributed random number $r$. If the GPI in this month is known, the GPI in the next month can be predicted by the actual GPI. If the GPI in this month is unknown, the GPI in the next month can be predicted by the predicted GPI of this month. The steps are recurred until the completion of construction.

5) Determination of completed period. The monthly GPIs obtained by the above GPI forecasting method are accumulated. When the accumulated GPI $H$ is not less than $100 \%$, the construction is completed. According to the formula, day $=(n-1)+(H-100) / S$, the project completion period can be obtained.

6) Completion probability in contract period. According to the predetermined number $N$, after simulation the number $Z$ is calculated, which indicates the completion period is not more than the contract period. The completion probability in the contract period is $Z / N$. The completion probability can reflect the project risk in general.

By Markov chain stimulation, the forecasting model of completion probability in contract period based on GPI can be obtained, which is illustrated in Figure 1.

Identifiers:

$m$ : repeated simulation times day: completion period;

$P(I, J)$ : transition probabilities $(I, J=1,2,3,4,5)$;

A: state matrix; $T$ : contract completion period;

$N$ : default maximum completion period;

$S$ : monthly GPI; $H$ : accumulated GPI;

p: completion probability in contract period.

\section{Project Application}

One steel enterprise is planning to construct a blast furnace of $4350 \mathrm{~m}^{3}$ in order to optimize the industrial structure and improve economic benefit. This project has been concluded into the national comprehensive programs. The project is designed to produce 3.5 million tons of molten iron, 1.153 million tons of granulating slag. Since it is a large-scale project, construction enterprise divides the whole into three sections. One certain Co. Ltd. has

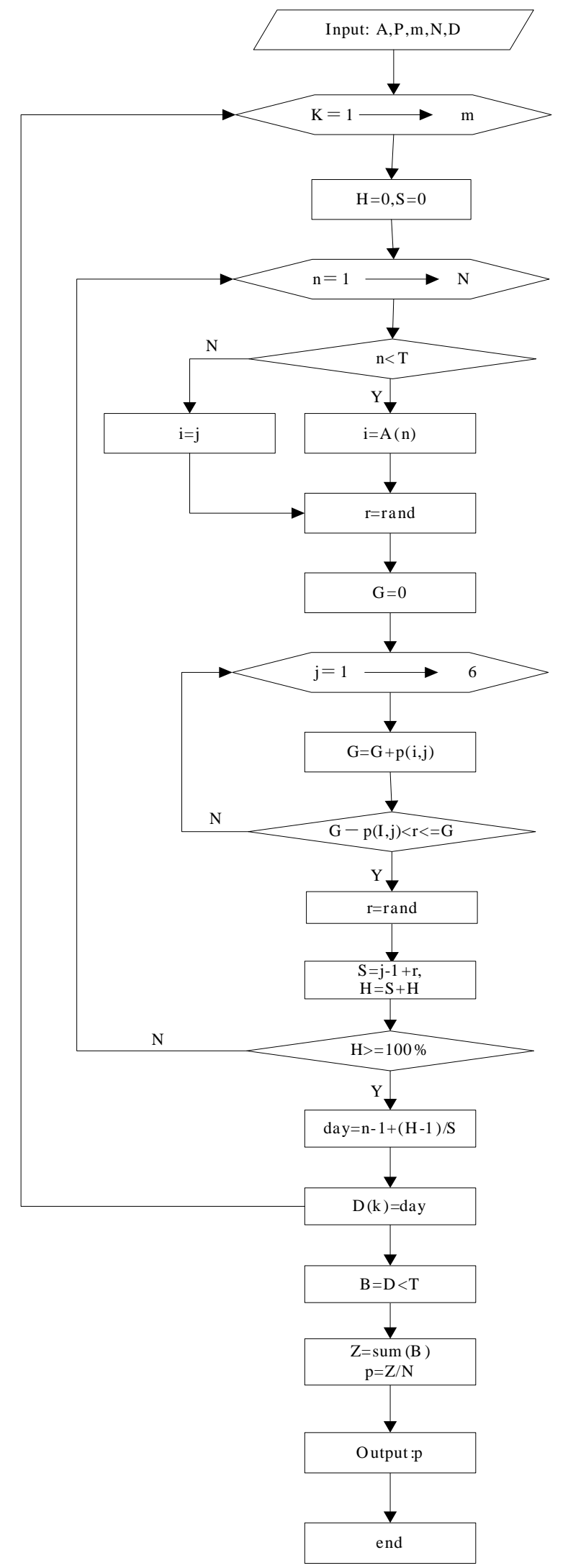

Figure 1. Block diagram of Markov chain stimulation model. 
Table 2. The GPI.

\begin{tabular}{|c|c|c|c|c|c|c|c|}
\hline Time & 2003.3 & 2003.4 & 2003.5 & 2003.6 & 2003.7 & 2003.8 & 2003.9 \\
\hline GPI & 1.06 & 1.2 & 1.45 & 1.22 & 2.42 & 2.5 & 3.09 \\
\hline Accumulatively GPI & 1.06 & 2.26 & 3.71 & 4.93 & 7.35 & 9.85 & 12.94 \\
\hline time & 2003.1 & 2003.11 & 2003.12 & 2004.1 & 2004.2 & 2004.3 & 2004.4 \\
\hline GPI & 3.27 & 4.21 & 4.23 & 4.46 & 4.87 & 5.02 & 5.03 \\
\hline Accumulatively GPI & 16.21 & 20.42 & 24.65 & 29.11 & 33.98 & 39 & 44.03 \\
\hline Time & 2004.5 & 2004.6 & 2004.7 & 2004.8 & 2004.9 & 2004.1 & 2004.11 \\
\hline GPI & 5.13 & 5.31 & 5.51 & 5.62 & 5.5 & 4.98 & 4.93 \\
\hline Accumulatively GPI & 49.16 & 54.47 & 59.98 & 65.6 & 71.1 & 76.08 & 81.01 \\
\hline time & 2004.12 & 2005.1 & 2005.2 & 2005.3 & 2005.4 & 2005.5 & 2005.6 \\
\hline GPI & 4.67 & 3.93 & 3.51 & 2.6 & 2.02 & 1.26 & 1 \\
\hline Accumulatively GPI & 85.68 & 89.61 & 93.12 & 95.72 & 97.74 & 99 & 100 \\
\hline
\end{tabular}

Note: the unit of each GPI is \%.

succeeded in contracting the construction of second and third sections. According to the contract made by two sides, the construction period is of 28 months, which is illustrated in Table 2.

Based on the established schedule predictive model, schedule controlling is done when it is in the simulation of Markov process. Corresponding program is organized in the use of MATLAB language, completing probability is $\mathrm{p}=85.56 \%$ of this project can be calculated, indicating the construction period of the project is with less risk. With the help of applying this model, it becomes more effective to control the progress and with more simple process.

\section{Conclusions}

This paper proposes GPI and its calculation method. The paper discusses that the transition of GPI from one moment to the next complies with Markov process. By MATLAB program, Markov stimulated process forecasting model has been established on the basis of GPI. The model, with its practicability and operability, provides a new approach, which facilitates the owner to control the process of large-scale projects macroscopically while overcoming the defects of traditional methods.

In the process of project construction, it is possible that the construction is completed ahead of or behind the schedule due to crashing or other causes. As this model can make forecast according to plan or actual GPI, it has great generality. This model can make forecast more realistic according to actual GPI in actual project control process. Furthermore, the constant renewal of forecast data fully shows that the project process control is dynamic.
The macro property of GPI determines that the data of model and forecast are macro. However, the data only make forecast from the perspective of time without taking cost and quality into consideration. Containing process, cost and quality, the integrated data will better serve the owner to realize the multi-objective integrated control of the project.

This study indicates that the owner only needs to focus on the GPI to control project process and to know project risk, which will not only make the management focus more prominent, but also can obtain more information. When forecast results show that the project risk is higher, the owner can promptly take corresponding measures to reduce the risk and to ensure project completion on schedule.

\section{REFERENCES}

[1] C. Hu, S. Liang and J. Wang, "A New Process Controlling Model of Construction Project and Its Application,” Journal of Xi' an University of Architecture \& Technology, Vol. 39, No. 4, 2007, pp. 468-473.

[2] C. Hu, D. Yu and S. Liang, "Process Control and Application of Large-Scale Metallurgical Project," Construction Technique, Vol. 34, No. 2, 2005, pp. 58-61.

[3] S. Liang, "Process Control and Forecasting Model of LargeScale Metallurgical Project,” Master Thesis, Xi'an University of Architecture \& Technology, Xi’an, 2006.

[4] X. Wang, “Random Process,” Xi'an Jiaotong University Press, Xi'an, 2001.

[5] X. Wang, "Study on Cost and Schedule Control of Large Scale Metallurgical Project," Master Thesis, Xi'an University of Architecture \& Technology, Xi'an, 2009.

[6] X. Xie, "Stimulation of Architectural Project System," Science Press, Beijing, 2001. 
Appendix: MATLAB Program

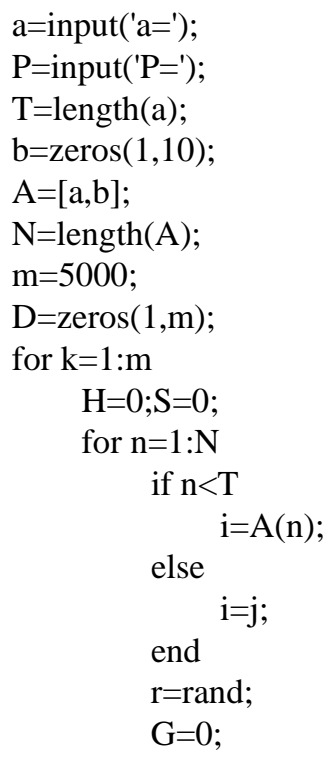

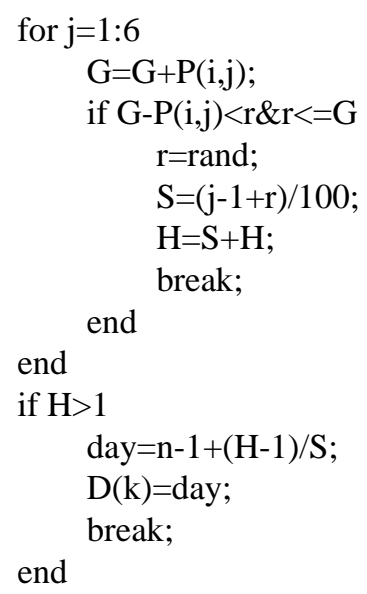

